\section{Potentiel thérapeutique de l'ambroxol contre les maladies du motoneurone}

Alexandra Bouscary, Cyril Quessada
Les sphingolipides : des acteurs majeurs dans la sclérose latérale amyotrophique

La sclérose latérale amyotrophique (SLA) est la maladie du motoneurone la plus fréquente chez l'adulte: environ 7000 personnes sont concernées par cette maladie en France. Cette maladie incurable est caractérisée par la mort sélective des neurones moteurs corticaux et des motoneurones bulbaires et spinaux, engendrant une paralysie progressive et le décès entre 2 et 5 ans après l'annonce du diagnostic. À ce jour, aucun traitement curatif n'est disponible contre cette maladie et les traitements symptomatiques ont une efficacité limitée. En France, seul un médicament, le Rilutek ${ }^{\circledR}$, est actuellement indiqué pour le traitement de la SLA : il permet d'augmenter l'espérance de vie de quelques mois.

De récentes études physiopathologiques ouvrent cependant la perspective d'identifier de nouvelles cibles thérapeutiques. Le métabolisme des lipides constitue une cible possible, car chez les patients atteints de SLA, l'incidence de la dyslipidémie et de l'hypermétabolisme (i.e., augmentation du métabolisme énergétique au repos) est plus forte, et l'existence d'un hypermétabolisme est associée à une progression plus rapide des symptômes de la maladie [1]. Les sphingolipides sont des lipides complexes, impliqués dans les voies de signalisation cellulaire, dans la maturation neuronale, dans la formation des gaines de myéline et donc dans la transmission du signal nerveux, ainsi que dans les processus de vieillissement [2]. De récentes études «métabolomiques » et «transcriptomiques » montrent que les principales classes de lipides, dont les sphingolipides, sont dérégulées chez les patients atteints de SLA. Ces altérations sont également retrouvées dans les modèles transgéniques murins de SLA avant l'apparition des premiers symptômes de la maladie $[3,4]$. Ce réarrangement du profil lipidique inclut notamment une stimulation des voies de dégradation du glucosylcéramide (Glc(er). Cette dérégulation pourrait jouer un rôle clé dans la physiopathologie de la SLA, et donc représenter une cible thérapeutique.

Pour étudier la contribution de ces perturbations dans le processus de neurodégénérescence de la SLA, nous avons utilisé des approches pharmacologiques. Ces approches ont consisté à réguler les niveaux de GlcCer en ciblant sa dégradation par les enzymes GBA et GBA2, deux $\beta$-glucocérébrosidases (GCase). En 2017, Henriques et collaborateurs ont montré une amélioration de la récupération fonctionnelle dans un modèle de compression nerveuse après l'administration de conduritol $B$ epoxyde ( $C B E)$, un inhibiteur irréversible des enzymes $\mathrm{GBA}$ et $\mathrm{GBA} 2$ [5]. Ce résultat suggère que le GlcCer contribue à la stabilité de l'unité motrice.

L'ambroxol : un candidat médicament Depuis 1970, l'ambroxol est un médicament générique expectorant et mucolytique utilisé dans le traitement des maladies inflammatoires des voies respiratoires (bronchite). L'ambroxol est l'un des dérivés synthétiques de la vasicine, substance active d'Adhatoda vasica, une plante anciennement
Inserm U1118, Mécanismes centraux et périphériques de la neurodégénérescence, Université de Strasbourg, France. Faculté de médecine, 11 rue Humann, 67085 Strasbourg, France.

alexandra.bouscary@gmail.com cyril.quessada@etu.unistra.fr

utilisée en Inde pour ces mêmes propriétés. L'ambroxol présente de nombreuses activités pharmacologiques. En effet, cet agent fluidifiant des sécrétions bronchiques limite la production de cytokines dans l'inflammation, protège contre le stress oxydant et présente des propriétés analgésiques par inhibition de certains canaux sodiques voltagedépendants impliqués dans la douleur [6]. Actuellement en vente libre dans la plupart des pays de l'Union Européenne, ce médicament a l'avantage de présenter un profil de sécurité favorable et un très faible risque d'effets secondaires. L'ambroxol est également décrit comme capable de moduler l'activité GCase [7] : cela pourrait être à l'origine d'un effet neuroprotecteur dans les maladies neurodégénératives ou de surcharge lysosomale, qui mérite d'être étudié.

L'utilisation de l'ambroxol relève d'une nouvelle stratégie thérapeutique pour corriger les défauts d'activité enzymatique, la «pharmacological chaperon therapy », qui consiste à utiliser une petite molécule capable d'entrer dans les cellules et de promouvoir ou restaurer l'activité d'une enzyme défaillante, par exemple pour empêcher une surcharge lysosomale. Cet agent pharmacologique capable de passer la barrière hématoencéphalique est envisagé comme traitement permettant de diminuer les niveaux de GlcCer dans la maladie de Parkinson et la maladie de Gaucher, deux maladies dans lesquelles on observe une accumulation toxique de GlcCer dans le système nerveux, due à un déficit d'activité enzymatique de GBA. La stratégie thérapeutique vise à utiliser l'ambroxol comme une molécule chaperonne pour 
A

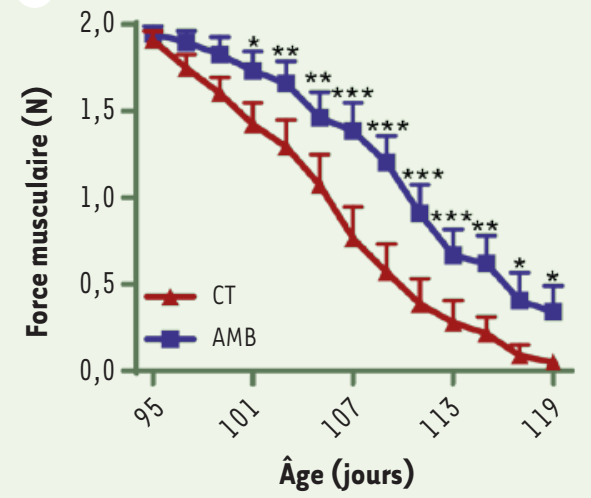

B

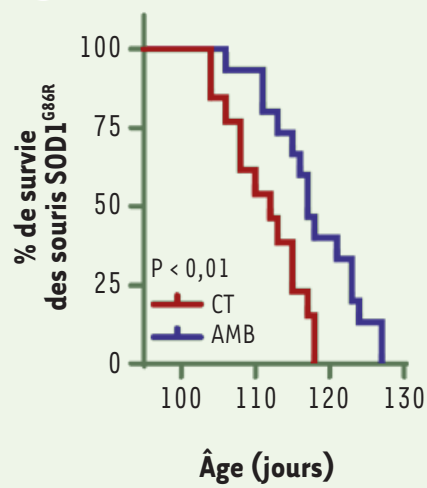

Figure 1. Effet protecteur de l'ambroxol sur le développement de la SLA dans le modèle murin SOD1 ${ }^{\text {G86R. }}$. Les souris ont été traitées soit par l'ambroxol (AMB) en eau de boisson soit à l'eau (contrôle, CT) à partir d'un âge où la maladie est symptomatique ( 95 jours). A. Le traitement par $A M B$ ralentit la perte de la force musculaire des souris ( $n=12$ à 15 par groupe; two-way ANOVA, $\left.{ }^{*} p<0,05 ;{ }^{*} p<0,01 ; * \star * p<0,001\right)$. B. Le traitement par AMB augmente la survie des souris de 6 jours ( $n=12$ à 15 par groupe; test non paramétrique du logrank, $p<0,01$ ) (figure adaptée de [11]).

restaurer l'activité enzymatique de GBA en améliorant son adressage vers le lysosome [8]. L'efficacité de l'ambroxol a été démontrée dans la maladie de Gaucher, où son utilisation a permis non seulement d'augmenter l'activité enzymatique de GBA, mais aussi d'améliorer les symptômes cliniques des patients [9]. De plus, un essai clinique de phase II mené avec des patients atteints de la maladie de Parkinson a démontré l'innocuité de la molécule à forte dose [10].

$\varepsilon n$ plus de son activité chaperonne sur GBA, l'ambroxol a la capacité d'inhiber I'activité enzymatique de GBA2 : un effet que nous avons utilisé pour tenter de traiter les souris SOD ${ }^{\text {G86R }}$, un modèle murin de la SLA par introduction d'une mutation dans le gène de la superoxyde dismutase 1 . Chez ces souris, nous avions mis en évidence une augmentation de $G B A 2$ dans la moelle épinière. Alors que GBA est une GCase du lysosome, GBA2 agit dans le réticulum endoplasmique et à la membrane plasmique. Nous avons montré que l'administration d'ambroxol a des effets bénéfiques sur l'évolution de la maladie et la durée de vie des animaux (Figure 1). Dans ce modèle animal de la SLA, l'ambroxol ralentit la destruction des jonctions neuromusculaires, préservant ainsi les muscles de la dénervation. De plus, aussi bien in vitro qu'in vivo (dans le cadre d'un modèle de compression nerveuse), nous avons montré que l'ambroxol stimule la plasticité axonale et la formation des jonctions neuromusculaires [11].

Malgré la dérégulation du métabolisme du GlcCer dans la SLA, cette maladie n'est pas caractérisée par une accumulation toxique de GlcCer dans les lysosomes. L'intérêt de l'utilisation de l'ambroxol dans la SLA est fondé sur I'hypothèse selon laquelle l'inhibition de GBA2, favoriserait l'interaction des sphingolipides de la membrane plasmique avec des facteurs neurotrophiques qui sont responsables de la croissance et de la survie des neurones. $\varepsilon$ effet, des études suggèrent que certains sphingolipides comme les gangliosides, dont le GlcCer est le précurseur, sont capables de potentialiser les neurotrophines et leurs récepteurs [12]. II est donc possible que les effets bénéfiques de l'ambroxol dans les maladies neurodégénératives résident aussi dans sa capacité à stimuler ou interagir avec ces facteurs neuroprotecteurs.
L'ensemble de nos résultats a mis en lumière des propriétés neuroprotectrices de l'ambroxol, jusqu'alors inconnues, dans un modèle murin de la SLA. Associés aux résultats obtenus par d'autres équipes de recherche [3], nos résultats incitent à tester l'ambroxol comme médicament candidat pour le traitement de la SLA. La disponibilité de l'ambroxol sur le marché du médicament pourrait faciliter la mise en place rapide d'essais cliniques. $\diamond$ Therapeutic potential of ambroxol for motor neuron diseases

\section{LIENS D'INTÉRÊT}

Les auteurs déclarent n'avoir aucun lien d'intérêt concernant les données publiées dans cet article.

\section{RÉFÉRENCES}

1. Jésus P, Fayemendy P, Nicol M, et al. Hypermetabolism is a deleterious prognostic factor in patients with amyotrophic lateral sclerosis. Eur J Neurol 2018 ; 25 : 97-104.

2. Olsen ASB, Færgeman NJ. Sphingolipids: membrane microdomains in brain development, function and neurological diseases. Open Biol $2017 ; 7$ : 170069.

3. Dodge JC, Treleaven CM, Pacheco J, et al. Glycosphingolipids are modulators of disease pathogenesis in amyotrophic lateral sclerosis. Proc Natl Acad Sci USA 2015; 112 : 8100-5.

4. Henriques A, Croixmarie V, Bouscary A, et al. Sphingolipid metabolism is dysregulated at transcriptomic and metabolic levels in the spinal cord of an animal model of amyotrophic lateral sclerosis. Front Mol Neurosci 2018 ; 10.

5. Henriques A, Huebecker M, Blasco H, et al. Inhibition of $\beta$-glucocerebrosidase activity preserves motor unit integrity in a mouse model of amyotrophic lateral sclerosis. Sci Rep 2017 ; 7.

6. Malerba M, Ragnoli B. Ambroxol in the $21^{\text {st }}$ century: pharmacological and clinical update. Expert Opin Drug Metab Toxicol 2008 ; 4 : 1119-29.

7. Shanmuganathan M, Britz-McKibbin P. Inhibitor screening of pharmacological chaperones for lysosomal $\beta$-glucocerebrosidase by capillary electrophoresis. Anal Bioanal Chem 2011 ; 399 : 2843-53.

8. Maegawa GHB, Tropak MB, Buttner JD, et al. Identification and characterization of ambroxol as an enzyme enhancement agent for Gaucher disease. J Biol Chem 2009 ; 284 : 23502-16.

9. Narita A, Shirai K, Itamura S, et al. Ambroxol chaperone therapy for neuronopathic Gaucher disease: a pilot study. Ann Clin and Transl Neurol 2016 ; 3 : 200-15.

10. Mullin S, Smith L, Lee K, et al. Ambroxol for the treatment of patients with Parkinson disease with and without glucocerebrosidase gene mutations: a nonrandomized, noncontrolled trial. JAMA Neurol 2020 ; doi: 10.1001/jamaneurol.2019.4611

11. Bouscary A, Quessada C, Mosbach A, et al. Ambroxol hydrochloride improves motor functions and extends survival in a mouse model of familial amyotrophic lateral sclerosis. Front Pharmacol $2019 ; 10: 883$.

12. Ledeen R, Wu G. Gangliosides of the nervous system. In : Sonnino S, Prinetti A, eds. Gangliosides. New York, NY : Springer, 2018 : 19-55. 